\title{
Pre-operative axillary staging: should core biopsy be preferred to fine needle aspiration cytology?
}

\author{
Raghavan Vidya', Fahad Mujtaba Iqbal' ${ }^{2}$ and Bernadette Bickley ${ }^{3}$ \\ ${ }^{1}$ County Hospital, Weston Road, Stafford ST16 3SA, UK \\ ${ }^{2}$ Keele University, David Weatherall Building, Keele University, Stoke-on-Trent, Staffordshire ST5 5BG, UK \\ ${ }^{3}$ County Hospital, Weston Road, Stafford ST16 3SA, UK
}

Correspondence to: Raghavan Vidya. E-mail: raghavan.vidya@nhs.net

\begin{abstract}
Objective: To determine the diagnostic accuracy of ultrasound guided fine needle aspiration (FNA) cytology and core needle biopsy (CNB) of axillary lymph nodes pre-operatively in newly diagnosed operable primary breast cancer.

Methods: An observational study for all patients who underwent pre-operative FNA cytology or CNB during September 2013-August 2014 was conducted at our institution (County Hospital, Stafford, UK). The accuracy of pre-operative axillary staging was compared to the postoperative histology. For this sensitivity, specificity, positive predictive value (PPV) and negative predictive values (NPV) were calculated.

Results: A total of 81 consecutive patients were evaluated by axillary ultrasound. Patients identified with potentially abnormal axillary lymph nodes underwent definitive surgery. Seven patients had positive cytology/histology who did not undergo definitive surgery and were excluded $(N=74)$ from the study. CNB had a sensitivity of $100 \%$ versus $72 \%(p=0.006)$ for FNA cytology. Both had $100 \%$ specificity and PPV. The NPV of CNB was $100 \%$ versus $72 \%$ for FNA cytology. Among $35 \%$ of patients that underwent FNA cytology required repeat procedure versus $2.6 \%$ of patients who underwent CNB. $0 / 38$ patients that had CNB required a second operation while $7 / 43$ patients with negative FNA cytology had positive lymph nodes identified at sentinel lymph node biopsy (SLNB) requiring surgical re-intervention with axillary node clearance.
\end{abstract}

Conclusion: CNB was superior to FNA cytology when interrogating the axilla. We recommend CNB to be adopted routinely in pre-operative axillary staging to reduce surgical re-intervention.

Keywords: preoperative staging, sensitivity, core needle, fine needle aspiration, breast

Published: 07/03/2017

Received: 09/10/2016

ecancer 2017, 11:724 DOI: 10.3332/ecancer.2017.724

Copyright: (c) the authors; licensee ecancermedicalscience. This is an Open Access article distributed under the terms of the Creative Commons Attribution License (http://creativecommons.org/licenses/by/3.0), which permits unrestricted use, distribution, and reproduction in any medium, provided the original work is properly cited. 


\section{Introduction}

Lymph node status is one of the most significant prognostic factors for patients with breast cancer [1]. Sentinel lymph node biopsy (SLNB) has become the standard of care in predicting the status of remaining lymph nodes with surgical intervention as we see axillary lymph node clearance occurring in lymph node positive patients [2]. Axillary lymph node clearance negatively affects patient quality of life (QoL) with significant morbidity [3].

In order to eliminate unnecessary axillary lymph node clearance, preoperative axillary staging using ultrasound (US) is routinely performed in all newly diagnosed breast cancer patients with reported specificities of $100 \%$ [4]. Identification of metastatic spread to the lymph nodes prior to surgery using US-guided fine needle aspiration (FNA) cytology or core needle biopsy (CNB) is of great importance for accurate staging and for reducing the need for SLNB [5].

The aim of this study was to determine the diagnostic accuracy of US-guided FNA cytology and CNB of axillary lymph node pre-operatively in newly diagnosed operable primary breast cancer.

\section{Methods}

This was approved by our local audit department. A total of 170 consecutive patients from September 2013-August 2014 with newly diagnosed breast cancer who had an axillary ultrasound (US) were reviewed. Abnormal or suspicious axillary lymph nodes were identified in 81 patients (47.6\%) and were included in the study. Axillary US was normal in 89 patients (52.4\%) who were excluded from the study.

An abnormal axillary node was biopsied using FNA cytology or CNB. Ultrasound-guided FNA cytology was performed utilising a 21-guage needle and CNB using a 14-guage automated biopsy device (Monopty, Bard Radiology, Covington, Georgia, USA). Abnormal axillary node on US was defined as: i) entirely hypoechoic node, ii) a node cortex $>2 \mathrm{~mm}$, iii) eccentric cortical lobulation, iv) a short-long axis ratio $>0.5$, v) a short axis $>10 \mathrm{~mm}$, or vi) peripheral nodal vascularity.

In cases with more than one suspicious node, FNA cytology or CNB was performed on the node demonstrating the most suspicious features. The radiologists performed FNA cytology when the lymph node was high lying, close to a blood vessel, or to the chest wall. Preoperative biopsy reports were compared with the histology following surgery. Pre-operatively when lymph node biopsy was negative, the gold standard for sentinel node detection was the 'combined technique' which used both the blue dye and radioisotope injection. In patients who preoperatively had positive lymph nodes, standard axillary clearance was carried out. The histology reports were retrieved from a pathology lab computer system.

Statistical analysis was performed with GraphPad (GraphPad Software, La Jolla California, USA). The sensitivity, specificity, positive predictive value (PPV) and negative predictive values (NPV) were calculated. A Fisher's exact test was calculated with $p<0.05$ defined as statistically significant.

\section{Results}

A total of 81 patients had preoperative biopsy (CNB, $n=38$; FNA cytology, $n=43)$. The results are shown in Figure 1: seven patients had positive cytology/histology that did not undergo definitive surgery and hence were excluded from the analysis ( $\mathrm{N}=74)$.

43 patients $(58.1 \%)$ underwent FNA cytology of which $15(34.8 \%)$ patients required a repeat procedure $(n=11$ with technically inadequate cytology; $n=4$ with suspicious cytology). In contrast, 38/74 (51.3\%) patients underwent CNB with $1 / 38$ (2.6\%) requiring a repeat procedure $(n=1$, a pathological node on US with benign histological changes demonstrating evidence of haemorrhage within the node, a repeat CNB because of the discordant result revealed malignant histological features).

True positives (TP) were cases with evidence of metastatic disease at CNB with metastatic deposits proven at axillary lymph node clearance (Figure 1, $n=27$ ). True negatives $(T N)$ were cases with negative histology for malignancy at CNB and were free of metastasis 
according to the gold standard SLNB (Figure 1, $\mathrm{n}=11$ ). False negatives $(F N)$ had negative histology for malignancy at CNB but were found to have metastasis at SLNB. There were no false negatives in our study. False positives (FP) had positive histology at CNB but were free of metastasls at axillary lymph node clearance, again, we had no FP $(n=0)$. Sensitivity $(28 \div(28+0))$, specificity, PPV, and NPV for CNB were $100 \%$ (Table 1).

TP were cases with evidence of metastatic disease identified on FNA positive cytology with metastatic deposits proven at axillary lymph node clearance (Figure 1, $n=18$ ). TN were cases with negative cytology for malignancy at FNA cytology and were free of metastasis according to the gold standard SLNB (Figure 1, $n=25$ ). FN were cases with negative cytology for malignancy at FNA cytology but had metastasis at SLNB $(n=7)$. These patients had surgical re-intervention as axillary lymph node clearance. FP were cases with positive cytology at FNA cytology but were free of metastasis at axillary lymph node clearance. There were no false positives in this study. Sensitivity and NPV were $72 \%$; specificity and PPV were $100 \%$.

TP were the cases with evidence of metastatic disease on FNA cytology/CNB that had metastatic deposits proven at axillary lymph node clearance $(n=46)$. TN were those cases with negative cytology or histology for malignancy and were free of metastasis according to the gold standard SLNB $(n=28)$. FN were cases with negative cytology or histology for malignancy but at SLNB where found to have metastasis $(n=7)$. These patients then proceeded to have axillary lymph node clearance. FP were cases with positive cytology or histology and free of metastasis at axillary lymph node clearance; there were no false positives in our study. CNB was more sensitive than FNA cytology and was statistically significant $(p=0.006)$.

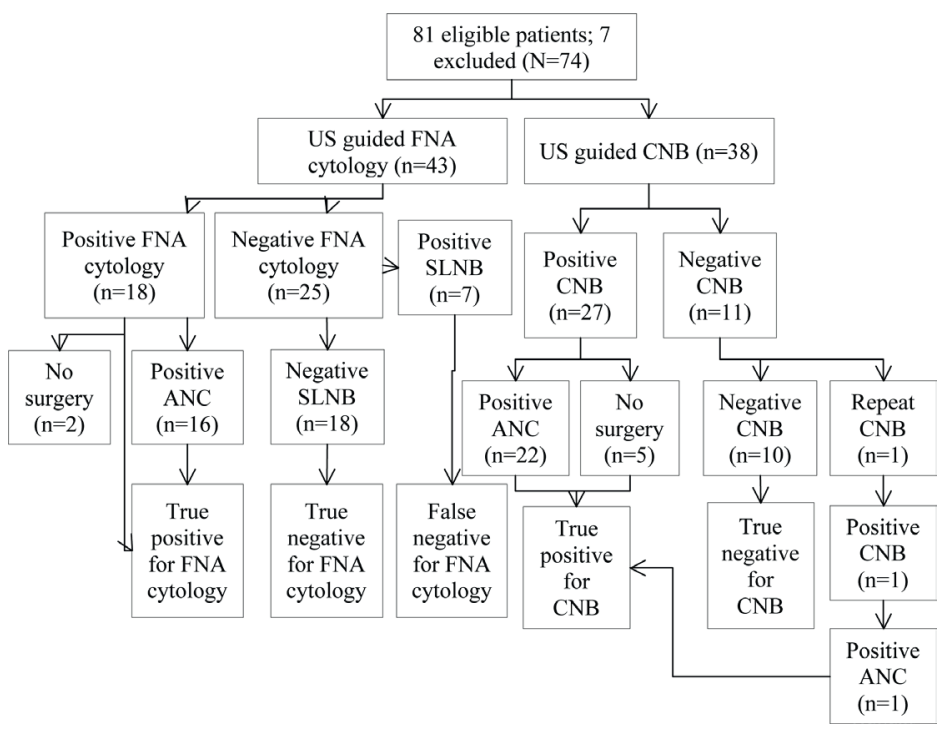

Figure 1. Flowchart of patients. US: ultrasound, CNB: core needle biopsy, FNA: fine needle aspiration, ANC: axillary lymph node clearance.

Table 1. Diagnostic performance of CNB and FNA cytology.

\begin{tabular}{|l|c|c|}
\hline \multicolumn{1}{|c|}{ Measure } & CNB (\%) & $\begin{array}{c}\text { FNA cytology } \\
\text { (\%) }\end{array}$ \\
\hline Sensitivity & 100 & 72 \\
\hline Specificity & 100 & 100 \\
\hline Negative predictive value & 100 & 72 \\
\hline Positive predictive value & 100 & 100 \\
\hline
\end{tabular}

CNB: core needle biopsy, FNA: fine needle aspiration 


\section{Discussion}

We demonstrated that patients in our series undergoing US-guided FNA cytology as a first line investigation are more likely to require a repeat procedure prior to their definitive surgery. Around $34.8 \%$ of patients that underwent this as a first line investigation required a repeat procedure compared to $2.63 \%$ of patients that had a US-guided CNB as their first line investigation. The use of US alone has a reported sensitivity of $61.4 \%$ (95\% confidence intervals $50.7-71.2)$; as a result we focused on the combined accuracy of US with tissue sampling [6].

A study in 2012 reported a similar specificity and PPV of $100 \%$ ( $n=210$ ) for US-guided CNB which was similar to our findings, but reported a significantly lower sensitivity (52.4\% versus $100 \%)$ [7]. This could be partly explained by differing tumour grades. A higher grade is more likely to be detected (sensitivities of $61 \%$ for grade 3 malignancies versus $35 \%$ for grade 1, p $=0.0146$ ) [7]. However, the grading in our series was not recorded and perhaps this count could account for the lower sensitivity. In addition, differences in operator's experience could also explain the lower sensitivity in their series. Higher sensitivities for CNB have been reported, a prospective study observed higher sensitivities for CNB versus FNA cytology $(88.2 \%$ versus $72.5 \%, p=0.008)$ with $100 \%$ specificity for both [6]. A significant difference between the sensitivity and NPV of CNB and FNA cytology were noted in our study. None of the 38 patients that had CNB sampling of their lymph nodes required a second operation $(F P=0)$. However, seven patients $(7 / 43)$ that had negative cytology at FNA cytology had positive lymph nodes identified at SLNB and therefore required a second surgical procedure $(p=0.0066)$.

In contrast, a meta-analysis found no significant difference in sensitivity between the two (FNA cytology $72.2 \%$, 95\% Cl 63.9-79.3 versus CNB 83.3\%, 95\% Cl 70.0-91.4) [8]. However, the studies incorporated in the analysis had differing US criteria for identifying suspicious lymph node lesions, thus potentially introducing significant heterogeneity. These findings were supported in a later study which favoured FNA cytology for financial reasons [8]. However, high FP for FNA cytology have been identified as a potential pitfall, although our study did not reproduce these findings [9, 10]. Inadequate sampling of FNA cytology is another pitfall that has been identified [9, 11]. Repeated sampling and a lower sensitivity could result in inadequately treated breast cancer.

In 2014, a review $(n=10,934)$ reported the accuracy of preoperative US and CNB for axillary staging in invasive breast cancer on two meta-analyses [12]. The use of US alone $(n=4313)$ gave a median sensitivity $61.4 \%$ (interquartile range [IQR] $51.2-79.4 \%)$ and median specificity of $82 \%$ (IQR, 76.9-89.0\%). Further analysis $(n=2805)$ of the accuracy of CNB established a pooled sensitivity of $79.6 \%(95 \%$ $\mathrm{Cl} 74.1-84.2 \%)$ and a high pooled specificity of $98.3 \%(95 \% \mathrm{Cl} 97.2-99 \%)$. This study also reported a similar PPV to ours (100\%, 95\% Cl 100-100\%) but a lower NPV $(67.4 \%, 95 \% \mathrm{Cl} 60-76.2 \%)$ [13]. In another meta-analysis, the combination of US with selective CNB $(n=9212)$ reported a pool sensitivity of $50 \%(95 \% \mathrm{Cl} 43-57 \%)$ with a FN rate of $25 \%(95 \% \mathrm{Cl} 24-27 \%)$ [14]. However, it should be noted that there was significant heterogeneity amongst the studies, making meaningful comparisons difficult. Some of which is likely to be attributed to the fact that such procedures are highly operator dependent.

A study in 2016 looked at all cases with newly diagnosed ipsilateral primary breast cancer that underwent axillary US-guided biopsies in a two year period with outcomes compared to the final histopathology from SLNB or ANC. They found there was a correlation towards CNB but no statistically significant in favour of either technique [15]. Similarly, a recent review reported no absolute superiority of CNB over FNA cytology and stated that cytopathologist experience is likely to influence the reported differences in the procedures [16]. Indeed, this suggests that operator skill is likely to play a large role.

A retrospective analysis reported a sensitivity for CNB of $69.1 \%$ and specificity of $100 \%(n=650)$; as a result, SLNB was avoided in $33 \%$ of patients [17]. CNB reduces the need for a second procedure which is beneficial to both the patient (reduced anxiety, reduced discomfort, fewer hospital visits) and the department.

In 2017, a meta-analysis ( $n=1082$ patients across 12 articles) reported a lower pooled sensitivity of $74 \%$ (95\% Cl 72-77\%for FNA cytology compared to the pooled sensitivity of CNB (87\%; 95\% Cl 84-88\%). However, there was significant heterogeneity for both analyses (I²0ver $88 \%$ ) implying that some caution should be used with interpreting the results. They reported similar specificities between CNB and FNA cytology (98\%; 95\% Cl 96\%-99\%, I²=76.2\% versus 96\%; 95\% Cl, 94-98\%, $\left.\mathrm{I}^{2}=39.0 \%\right)$ [18]. Again, the high heterogeneity particularly for CNB results should be considered. Nevertheless, they reported that CNB has a superior sensitivity to FNA cytology. 
There are limitations to our study as axillary US and tissue sampling are operator dependent techniques and this may have influenced our results. Secondly, tumour characteristics and patient demographics were not looked at in this study. The sensitivity for preoperative US-guided FNA cytology of abnormal lymph nodes in invasive ductal carcinoma has been shown to be high but not so for invasive lobular carcinoma (98.4\% versus $53.6 \%, p<0.001, n=142$ axillae) [19]. This suggests that FNA cytology may not be a suitable method in all patients for preoperative axillary assessing in invasive lobular carcinoma. Therefore, these factors may have influenced the accuracy of US findings and node positive rates in our study. Lastly, our study focused on US guided tissue sampling techniques and hence only consecutive cancer patients who had US scans were included which may have introduced a selection bias.

\section{Conclusion}

We found US-guided CNB to be a superior interventional technique when interrogating the axilla in our series. It is therefore recommended to perform a CNB as the first line investigation of potentially abnormal axillary lymph nodes, wherever possible, in order to reduce the likelihood of the patient requiring a second procedure whilst also improving sensitivity and NPV. Axillary US-guided CNB remains a valuable triage test for preoperative staging of the axilla as it helps to identify those patients unsuitable for SLNB. A large double-blind randomised controlled trial is needed for establishing whether any relationships exist between tumour characteristics and patient demographics and accuracy of US and lymph node sampling. Larger studies are also needed to quantify the role of the operator's experience in performing these investigations with patients blinded to having either US guided CNB or FNA cytology.

\section{List Of Abbreviations}

CNB: core needle biopsy

FNA: fine needle aspiration

PPV: positive predictive value

NPV: negative predictive value

US: ultrasound

TP: true positives

TN: true negatives

$\mathrm{FN}$ : false negatives

SLNB: sentinel lymph node biopsy

FP: false positives

ANC:axillary lymph node clearance.

\section{Conflicts of Interest}

The authors report no conflicts of interest.

\section{References}

1. Stavros, AT. Breast Ultrasound. 1st ed. Philadelphia, PA: Lippincott Williams \& Wilkins (2004).

2. Dixon M (1998) Sentinel node biopsy in breast cancer: a A promising technique, but it should not be introduced without proper trials BMJ Br Med J 317(7154) 295-6 DOI: 10.1136/bmj.317.7154.295 
3. Carlson GW and Wood WC (2011) Management of axillary lymph node metastasis in breast cancer: making progress JAMA 305(6) 606-7 DOI: 10.1001/jama.2011.131 PMID: 21304087

4. Cools-Lartigue $\mathrm{J}$ and Meterissian S (2012) Accuracy of axillary ultrasound in the diagnosis of nodal metastasis in invasive breast cancer: a review World J Surg 36(1) 46-54 DOI: 10.1007/s00268-011-1319-9

5. Genta $\mathrm{F}$ et al (2007) Cost/accuracy ratio analysis in breast cancer patients undergoing ultrasound-guided fine-needle aspiration cytology, sentinel node biopsy, and frozen section of node World J Surg 31(6) 1157-65 DOI: 10.1007/s00268-007-9009-3

6. Rautiainen S et al (2013) Axillary lymph node biopsy in newly diagnosed invasive breast cancer: comparative accuracy of fine-needle aspiration biopsy versus core-needle biopsy Radiology 269(1) 54-60 DOI: 10.1148/radiol.13122637 PMID: 23771915

7. Solon JG et al (2012) Ultrasound-guided core biopsy: an effective method of detecting axillary nodal metastases $J$ Am Coll Surg 214(1) 12-7 DOI: 10.1016/j.jamcollsurg.2011.09.024

8. Ahn HS et al (2013) Comparison of sonography with sonographically guided fine-needle aspiration biopsy and core-needle biopsy for initial axillary staging of breast cancer J Ultrasound Med 32(12) 2177-84 PMID: 24277901

9. Ciatto $\mathrm{S}$ et al (2007) Accuracy of fine needle aspiration cytology (FNAC) of axillary lymph nodes as a triage test in breast cancer staging Breast Cancer Res Treat 103(1) 85-91 DOI: 10.1007/s10549-006-9355-0

10. Kuenen-Boumeester $V$ et al (2003)Ultrasound-guided fine needle aspiration cytology of axillary lymph nodes in breast cancer patients. A preoperative staging procedure Eur J Cancer 39(2) 170-4 DOI: 10.1016/S0959-8049(02)00501-4 PMID: 12509948

11. Jung $\mathrm{J}$ et al (2010) Accuracy of preoperative ultrasound and ultrasound-guided fine needle aspiration cytology for axillary staging in breast cancer ANZ J Surg 80(4) 271-5 DOI: 10.1111/j.1445-2197.2009.05090.x PMID: 20575955

12. Houssami $\mathrm{N}$ and Turner RM (2014) Staging the axilla in women with breast cancer: the utility of preoperative ultrasound-guided needle biopsy Cancer Biol Med 11(2) 69-77 PMID: 25009748 PMCID: 4069800

13. Houssami $\mathrm{N}$ et al (2011) Preoperative ultrasound-guided needle biopsy of axillary nodes in invasive breast cancer: meta-analysis of its accuracy and utility in staging the axilla Ann Surg 254(2) 243-51 DOI: 10.1097/SLA.0b013e31821f1564 PMID: 21597359

14. Diepstraten SE et al (2014) Value of preoperative ultrasound-guided axillary lymph node biopsy for preventing completion axillary lymph node dissection in breast cancer: a systematic review and meta-analysis Ann Surg Oncol 21(1) 51-9 DOI: $10.1245 / \mathrm{s} 10434-013-3229-6$

15. Zosimas D, Lykoudis PM and Vashisht $R$ (2016) Preoperative ultrasound guided percutaneous axillary biopsy in breast cancer patients: fine needle aspiration cytology versus core biopsy Ann Ital Chir 87 509-16 PMID: 27594687

16. Mitra $S$ and Dey $P(2016)$ Fine-needle aspiration and core biopsy in the diagnosis of breast lesions: a comparison and review of the literature Cytojournal 1318 DOI: 10.4103/1742-6413.189637 PMID: 27651820 PMCID: $\underline{5019018}$

17. Garcia-Ortega MJ et al (2011) Pretreatment axillary ultrasonography and core biopsy in patients with suspected breast cancer: diagnostic accuracy and impact on management Eur J Radiol 79(1) 64-72 DOI: 10.1016/j.ejrad.2009.12.011

18. Wang M et al (2017) A sensitivity and specificity comparison of fine needle aspiration cytology and core needle biopsy in evaluation of suspicious breast lesions: a systematic review and meta-analysis Breast 31 157-66 DOI: 10.1016/j.breast.2016.11.009

19. Topps A et al (2014) The sensitivity of pre-operative axillary staging in breast cancer: comparison of invasive lobular and ductal carcinoma Eur J Surg Oncol 40(7) 813-7 DOI: 10.1016/j.ejso.2014.03.026 PMID: 24797703 